\title{
Conserving the understudied invertebrates: a call for a systematic monitoring protocol for Asian horseshoe crabs in nursery habitats
}

\author{
Chun-Chieh Wang ${ }^{1,2,3}$, Shiang-Lin Huang ${ }^{2}$, Xueping Wang ${ }^{4}$, Peng $\mathrm{Xu}^{4}$, \\ Xing Huang ${ }^{4}$, Yongyan Liao ${ }^{3,4}$, Xiaoyong Xie ${ }^{5}$, Kit Yue Kwan ${ }^{3,4, *}$ \\ ${ }^{1}$ Marine Biology Institute, College of Science, Shantou University, Shantou, Guangdong, 515063, PR China \\ ${ }^{2}$ Guangxi Beibu Gulf Marine Research Center, Guangxi Academy of Sciences, Nanning, Guangxi, 530000, PR China \\ ${ }^{3}$ Guangxi Key Laboratory of Beibu Gulf Biodiversity Conservation, Beibu Gulf University, Qinzhou, Guangxi, 535011, PR China \\ ${ }^{4}$ College of Marine Sciences, Beibu Gulf University, Qinzhou, Guangxi, 535011, PR China \\ ${ }^{5}$ Key Laboratory of South China Sea Fishery Resources Exploitation \& Utilization, Ministry of Agriculture of China, \\ South China Sea Fisheries Research Institute, Chinese Academy of Fishery Sciences; Guangdong Provincial Key Laboratory of \\ Fishery Ecology and Environment, Guangzhou, Guangdong, 510300, PR China
}

\begin{abstract}
Baseline information on population size, trends and spatial distribution are important for population status assessments of threatened taxa. The 3 species of Asian horseshoe crabs (Tachypleus tridentatus, T. gigas, Carcinoscorpius rotundicauda) are declining throughout their geographic ranges, and identifying their essential nursery habitats and monitoring juvenile population changes is necessary for conservation management. We reviewed juvenile population studies published during 2001-2019 to evaluate which sampling methods were commonly adopted and how baseline data were presented. The applied methods included random or systematic quadrat $(35 \%)$, random visual search $(41 \%)$ and belt transect $(47 \%)$. Juvenile population sampling and data reporting were generally insufficient for assessing population status and clarifying nursery habitat configuration. We propose a conceptual framework and minimum standards for collecting and reporting juvenile population and nursery habitat baseline information. We also outline the importance of showing population density, rather than abundance, and spatial distribution in relation to habitat seascape or landscape satellite images. The framework and recommendations will improve the feasibility of robust, long-term monitoring of juvenile Asian horseshoe crab population status and nursery habitat, thus serving regional conservation management purposes.
\end{abstract}

KEY WORDS: Baseline $\cdot$ IUCN Red List · Assessment · Carcinoscorpius rotundicauda $\cdot$ Tachypleus gigas $\cdot$ Tachypleus tridentatus

\section{INTRODUCTION}

A robust monitoring program on distribution and population size of taxa is essential not only to ecological studies but also to the design and evaluation of effective conservation practices (Conway 2011, Per-

${ }^{*}$ Corresponding author: kityuekwan@bbgu.edu.cn rig et al. 2019). Baseline information, including an estimate of population size, growth and extent of occupancy, is necessary for population status assessment following IUCN Red List criteria (Gärdenfors et al. 2001). To collect relevant baseline data for evaluating population status, however, can be challenging

() The authors 2019. Open Access under Creative Commons by Attribution Licence. Use, distribution and reproduction are unrestricted. Authors and original publication must be credited. 
for widely distributed marine species, due to the information gap across the geographic range, and the incompatability of sampling methods. The lack of baseline data can impede the progress of effective conservation and management planning.

The 3 Asian horseshoe crab species, i.e. tri-spine Tachypleus tridentatus, mangrove Carcinoscorpius rotundicauda and coastal horseshoe crab T. gigas, are distributed widely across the subtropical and tropical waters from East Asia, including the Seto Inland Sea of Japan and the southeastern coast of China, through Southeast Asia to the Bay of Bengal (John et al. 2018, Liao et al. 2019a). Natural population overexploitation and shoreline reclamation have been identified as the main threats driving apparent population declines (Hsieh \& Chen 2015, John et al. 2018, Fu et al. 2019, Liao et al. 2019b). While C. rotundicauda and T. gigas are categorised as Data Deficient on the IUCN Red List; $T$. tridentatus has been listed as Endangered (Laurie et al. 2019). Direct species protection and indirect habitat protection measures at specific sites for Asian horseshoe crabs are limited to a few countries or regions. Long-term population monitoring and habitat baseline collection are thus essential to inform scientifically sound conservation management.

In this Note, we summarise the sampling methods and data presentation of juvenile Asian horseshoe crab population studies published during 2001-2019. Due to the differences in habitats used by horseshoe crabs throughout their ontogeny, we did not attempt to extrapolate the findings to feeding and spawning habitats of adults. We tabulate common pitfalls of sampling and data reporting that may hinder scientific information transmission, and propose a conceptual framework and minimum standards to guide future population monitoring and collecting of baseline habitat data on nursery grounds.

\section{MATERIALS AND METHODS}

Databases and search engines, mainly Web of Science, Scopus, China National Knowledge Infrastructure, Scholarly and Academic Information Navigator, Japan, and Google Scholar, were used to identify articles relevant to population studies and nursery habitats of Asian horseshoe crabs. Seven key words were used in combination: horseshoe crab, Tachypleus, Carcinoscorpius, juvenile, nursery, population, and habitat. Reference searching using local names of horseshoe crabs was also conducted using different languages to include more studies (Table S1 in the
Supplement at www.int-res.com/articles/suppl/n040 p369_supp.pdf). The retrieved data were screened manually to remove duplicate references. Publications in which horseshoe crabs were sampled, but which were not specifically designed as juvenile population studies, were excluded. We applied 20 detailed criteria in 3 categories (Table 1; Tables S2S4), including population baselines, nursery habitat baselines and sampling conditions, to assess whether the population studies contained all important information required for population status assessment and nursery habitat configuration.

\section{RESULTS}

We identified 17 published articles or book chapters that documented the juvenile population size, natural growth and nursery habitat conditions. The number of studies that used the 3 different sampling methods, i.e. random/systematic quadrat ( $35 \%$ ), random visual search $(41 \%)$ and belt transect $(47 \%)$, was similar (Table 1). Nearly half of the studies (47\%) sampled within $<5 \%$ of the total suitable habitat area or did not justify whether the sampling area was sufficient to represent the entire habitat (Table 1). Issues regarding the comparability of studies using different methods $(29 \%)$ and the presentation of data in terms of abundance instead of density $(12 \%)$ were also noted. Most studies included sampling condition information such as survey period (month) and season, number of transects and quadrats, area of quadrat, as well as length and distance between transects. However, information which informs survey efforts, such as sampling hour, number of surveyors and sampled shore length, was seldom included (Table 1).

\section{DISCUSSION}

The number of studies on quantifying juvenile population and habitat baselines to serve conservation management purposes has increased in recent years. However, important baselines and survey information are often not provided to a sufficient degree, thus limiting the practicality of long-term juvenile population monitoring at local nursery habitats. Considering the patchy distributions and considerable seasonality of juvenile horseshoe crabs on nursery grounds, direct comparisons between datasets collected from different locations and times may result in spurious conclusions and flawed management 
Table 1. Survey methods for populations of juvenile Asian horseshoe crabs, and the accompanying issues and deficiencies in reporting population and habitat baseline data

\begin{tabular}{|c|c|c|}
\hline & $\begin{array}{l}\text { Total } \\
\text { count }\end{array}$ & Percentage \\
\hline Issues in reporting population baselines & 17 & \\
\hline Data expressed as abundance (no. of individuals) ${ }^{a}$ & 2 & 11.8 \\
\hline Sampling area $<5 \%$ of the entire habitat/cannot be justified & 8 & 47.1 \\
\hline Comparing studies using different sampling methods & 5 & 29.4 \\
\hline Comparing studies with different life stages (juvenile vs. adult) & 1 & 5.9 \\
\hline Polling data from different sampling methods & 1 & 5.9 \\
\hline \multicolumn{3}{|l|}{ Deficiencies in reporting sampling methods } \\
\hline Random visual search & 7 & \\
\hline Nursery habitat baselines & & \\
\hline Sampling location/point coordinates & 2 & 28.6 \\
\hline Total suitable habitat area & 3 & 42.9 \\
\hline Sampling period/month/season & 0 & 0.0 \\
\hline Habitat conditions & 3 & 42.9 \\
\hline Sampling time relative to tidal level & 3 & 42.9 \\
\hline \multicolumn{3}{|l|}{ Sampling conditions } \\
\hline Total sampling area & 3 & 42.9 \\
\hline Sampling shore length & 5 & 71.4 \\
\hline Sampling hour & 1 & 14.3 \\
\hline No. of surveyors & 5 & 71.4 \\
\hline Random/systematic quadrat & 6 & \\
\hline \multicolumn{3}{|l|}{ Nursery habitat baselines } \\
\hline Sampling location/point coordinates & 1 & 16.7 \\
\hline Total suitable habitat area & 3 & 50.0 \\
\hline Sampling period/month/season & 0 & 0.0 \\
\hline Habitat conditions & 1 & 16.7 \\
\hline Sampling time relative to tidal level & 4 & 66.7 \\
\hline \multicolumn{3}{|l|}{ Sampling conditions } \\
\hline Sampling shore length & 4 & 66.7 \\
\hline No. of transects & 0 & 0.0 \\
\hline Transect length & 0 & 0.0 \\
\hline Distance between transects & 0 & 0.0 \\
\hline No. of quadrats per transect & 0 & 0.0 \\
\hline Area of each quadrat & 0 & 0.0 \\
\hline Belt transect & 8 & \\
\hline \multicolumn{3}{|l|}{ Nursery habitat baselines } \\
\hline Sampling location/point coordinates & 1 & 12.5 \\
\hline Total suitable habitat area & 6 & 75.0 \\
\hline Sampling period/month/season & 0 & 0.0 \\
\hline Habitat conditions & 2 & 25.0 \\
\hline Sampling time relative to tidal level & 5 & 62.5 \\
\hline \multicolumn{3}{|l|}{ Sampling conditions } \\
\hline Sampling shore length & 6 & 75.0 \\
\hline No. of transects & 0 & 0.0 \\
\hline Transect length & 0 & 0.0 \\
\hline Transect width & 0 & 0.0 \\
\hline Distance between transects & 1 & 12.5 \\
\hline Sampling hour & 7 & 87.5 \\
\hline No. of surveyors & 5 & 62.5 \\
\hline
\end{tabular}

decisions. We therefore propose a conceptual framework (Fig. 1) and recommendations (Table 2) for collecting and reporting juvenile population and habitat baseline data, based on the current review of existing literature published between 2001 and 2019.

Random visual searching within a predefined and representative area of nursery habitat is recommended for the collection of juvenile horseshoe crab occurrence data across wider geographic ranges (Weng et al. 2012). To sample juvenile populations in local nursery habitats, systematic surveys with transects and quadrats are encouraged (Hu et al. 2009, Shin et al. 2009, Kwan et al. 2016, Lee \& Morton 2016; see Fig. S1 as an example). Number and size of quadrats, as well as the suitability of applying randomly or regularly spaced transects should be assessed and decided for each nursery shore after conducting a pilot study. Environmental baselines, particularly surface water temperature and sediment physico-chemical parameters (e.g. grain size, total organic content and chlorophyll a), should be collected in parallel with juvenile population determination, as these parameters have been identified as important environmental factors correlating to juvenile densities (Hsieh \& Chen 2009, Morton \& Lee 2010, Kwan et al. 2016). Relative positions of mangrove forests, seagrass patches, tidal creeks and other freshwater inputs should also be considered when explaining habitat use by juvenile horseshoe crabs (Xie et al. 2019). To determine the spatial heterogeneity of juveniles in each habitat, we suggest reporting the statistical dispersion of the density data in addition to mean values, e.g. mean $\pm \mathrm{SD}$ or visualising the data in box plots. Apart from providing overall density data for monitoring long-term trends in population growth within a spe- 

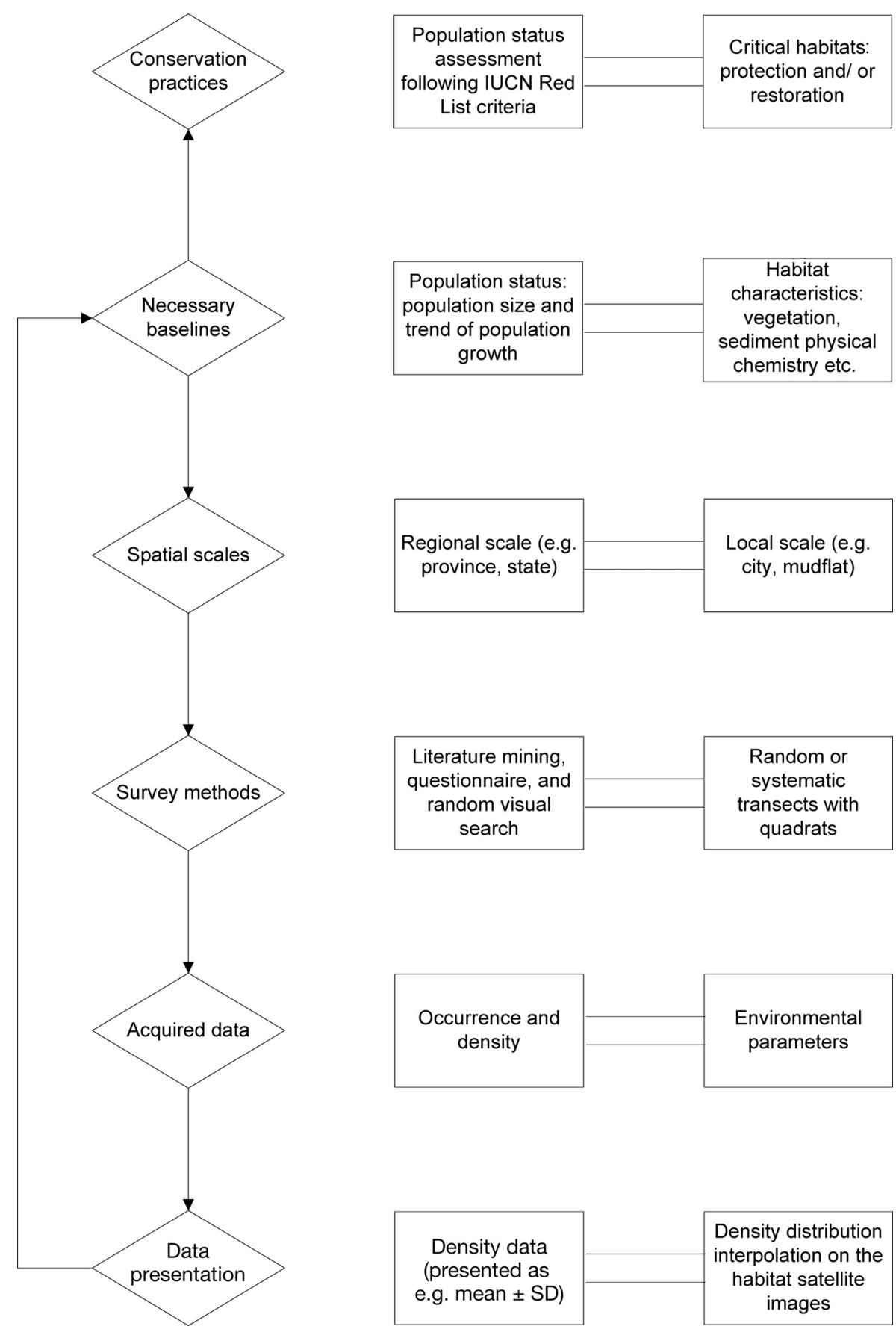

Fig. 1. Conceptual framework showing the structure of future studies which consider determination of baseline information, spatial scales and survey methods, collected data, data presentation, and application to conservation of Asian horseshoe crabs.

Parallel lines between boxes represent simultaneous consideration of the contents from the paired boxes

cific habitat, producing density distribution maps across the entire survey area (see Fig. S2 as an example) is useful to identify core habitat use areas (Xie et al. 2019). Such maps also allow visualising juvenile distribution patterns in relation to the surrounding landscape or seascape, such as the positions of river mouths, mudflats, mangrove forests and shoreline. The list of recommendations and the conceptual framework can be used as a guideline in future population studies to enhance the feasibility of scientifically robust baseline information collection and longterm monitoring. 
Table 2. Recommendations for collecting and reporting population and habitat baseline data for juvenile Asian horseshoe crabs

Recommendations

1. Consider the size of the sampled area relative to the total suitable habitat of a shore.

2. Evaluate the suitability of applying randomly or regularly spaced transects in preliminary studies.

3. Assess the number and size of applied quadrats along each transect in pilot trials.

4. Collect habitat baseline data, particularly water temperature and sediment physico-chemical parameters during juvenile population sampling.

5. Express the data in density instead of abundance (number of individuals).

6. Show the data dispersion of density (e.g. mean $\pm \mathrm{SD}$ ) rather than mean value only.

7. Visualise the spatial distribution of the juvenile population across the entire survey area.

8. Map the environmental characteristics across the entire survey area.

9. Consider the relative position of landscape or seascape such as mangrove forest, seagrass patch, tidal creek and other freshwater inputs within the survey area when explaining the habitat use distribution of juveniles.

10. Report the weather and tidal levels when sampling. Note the biological rhythm of juvenile emergence from the sediment.

11. Consider long-term habitat changes of the sampled shore.

Acknowledgements. This study was supported by the National Natural Science Foundation of China (41706183), Guangxi Natural Science Foundation (2017GXNSFBA198 181), Guangxi Recruitment Program of 100 Global Experts, and Guangxi BaGui Youth Scholars Programme. We are grateful to Anniqa C. K. Law for inspiring us to prepare this manuscript.

\section{LITERATURE CITED}

Conway CJ (2011) Standardized North American marsh bird monitoring protocol. Waterbirds 34:319-347

Fu Y, Huang S, Zhou W, Wang CC and others (2019) Sociodemographic drivers and public perceptions of consumption and conservation of Asian horseshoe crabs in northern Beibu Gulf, China. Aquat Conserv 29:1268-1277

Gärdenfors U, Hilton Taylor C, Mace GM, Rodríguez JP (2001) The application of IUCN Red List criteria at regional levels. Conserv Biol 15:1206-1212

Hsieh HL, Chen CP (2009) Conservation program for the Asian horseshoe crab Tachypleus tridentatus in Taiwan: characterizing the microhabitat of nursery grounds and restoring spawning grounds. In: Tanacredi JT, Botton ML, Smith DR (eds) Biology and conservation of horseshoe crabs. Springer, Boston, MA, p 417-438

Hsieh HL, Chen CP (2015) Current status of Tachypleus tridentatus in Taiwan for Red List assessment. In: Carmichael RH, Botton ML, Shin PKS, Cheung SG (eds) Changing global perspectives on horseshoe crab biology, conservation and management. Springer International Publishing, Cham, p 383-396

Hu M, Wang Y, Chen Y, Cheung SG, Shin PKS, Li Q (2009) Summer distribution and abundance of juvenile Chinese horseshoe crabs Tachypleus tridentatus along an intertidal zone in southern China. Aquat Biol 7:107-112

John BA, Nelson BR, Sheikh HI, Cheung SG and others (2018) Correction to: a review on fisheries and conservation status of Asian horseshoe crabs. Biodivers Conserv 27:3845

Kwan BKY, Hsieh HL, Cheung SG, Shin PKS (2016) Present population and habitat status of potentially threatened Asian horseshoe crabs Tachypleus tridentatus and Car-

Editorial responsibility: Nils Bunnefeld,

Stirling, UK cinoscorpius rotundicauda in Hong Kong: a proposal for marine protected areas. Biodivers Conserv 25:673-692

Laurie K, Chen CP, Cheung SG, Do V and others (2019) Tachypleus tridentatus (errata version published in 2019). The IUCN Red List of Threatened Species 2019: e.T21309A149768986. http://dx.doi.org/10.2305/IUCN. UK.2019-1.RLTS.T21309A149768986.en (accessed 13 Dec 2019)

Lee CNW, Morton B (2016) Changes in the distributions of juvenile horseshoe crabs (Arthropoda: Chelicerata) (2002-2014) related to environmental perturbations at Pak Nai and Ha Pak Nai, Deep Bay, Hong Kong SAR, China. Mar Pollut Bull 108:134-146

Liao Y, Liu K, Wu H, Xu Y, Huang H, Xu S, Kwan KY (2019a) How survival and food intake of Chinese horseshoe crabs, Tachypleus tridentatus respond to thermal variation: implications for understanding its distribution limit. J Nat Hist 53:1951-1960

Liao Y, Hsieh HL, Xu S, Zhong Q and others (2019b) Wisdom of crowds reveals decline of Asian horseshoe crabs in Beibu Gulf, China. Oryx 53:222-229

Morton B, Lee CN (2010) Spatial and temporal distributions of juvenile horseshoe crabs (Arthropoda: Chelicerata) approaching extirpation along the northwestern shoreline of the New Territories of Hong Kong SAR, China. J Nat Hist 45:227-251

* Perrig PL, Lambertucci SA, Donadio E, Padró J, Pauli JN (2019) Monitoring vultures in the $21^{\text {st }}$ century: the need for standardized protocols. J Appl Ecol 56:796-801

Shin PKS, Li HY, Cheung SG (2009) Horseshoe crabs in Hong Kong: current population status and human exploitation. In: Tanacredi JT, Botton ML, Smith DR (eds) Biology and conservation of horseshoe crabs. Springer, Boston, MA, p 347-360

Weng ZH, Xie YJ, Xiao ZQ, Huang LM, Li J, Wang SH, Zhang YZ (2012) Distribution and resource of Chinese horseshoe crab (Tachypleus tridentatus) in Fujian and other coast water of China. Chin J Zool 47:40-48 (in Chinese with English Abstract)

*Xie X, Wu Z, Wang CC, Fu Y and others (2019) Nursery habitat for Asian horseshoe crabs along the northern Beibu Gulf, China: implications for conservation management under baseline gaps. Aquat Conserv: Mar Freshw Ecosyst https://doi.org/10.1002/aqc.3259

Submitted: August 13, 2019; Accepted: October 24, 2019 Proofs received from author(s): December 13, 2019 